\title{
Dimensions of the semantic differential as cues in discrimination learning
}

\author{
D. M. McCORD and P. S. SIEGEL \\ University of Alabama, University, Alabama 35486
}

\begin{abstract}
The present study addresses two questions: (1) Will Osgood's semantic differential dimensions, activity and potency, provide adequate cuing to the human learner in a binary choice selective (discrimination) learning paradigm? And (2) will these dimensions combine as redundant cues to facilitate learning? Undergraduate student subjects responded to a program presented by microcomputer. Three groups were designated: Group $P$ was presented word pairs that differed only in their factor scores on potency (weak-strong); the other semantic dimensions were "neutralized." Group A received word pairs differing only in activity scores (active-passive). And Group A\&P-matched received word pairs differing in both potency and activity (relevant redundant cues, as weak-passive vs. strong-active). Instructions to all subjects simply urged that they respond to the "way the word feels-the general feeling of the word." Few subjects mastered the one dimension problem (potency or activity presented singly). Combining the two, however, yielded evidence of learning.
\end{abstract}

The simultaneous two-choice discrimination learning paradigm has long held an important place in experimental psychology. Utilized by early learning theorists such as Lashley, Krech, and Spence, this paradigm continues to serve as a major tool in the investigation of discrimination (selective) learning. The recent theories of Fisher and Zeaman (1973), Kendler and Kendler (1962), Sutherland and Mackintosh (1971), and Trabasso and Bower (1968) have, at some point in their development, relied upon data from two-choice discrimination learning.

One of the most robust findings within the discrimination learning literature is the facilitatory effect of presenting redundant relevant cues, the so-called RRC effect (Bourne \& Haygood, 1959; Trabasso \& Bower, 1968). With rare exception, redundant relevant cues facilitate learning when compared with single-dimension arrangements. Because of the high reliability of this finding, a primary obligation of any broad-scope theory of discrimination learning is that of explaining just how this facilitation comes about.

As cues, most investigations of discrimination learning have utilized geometric shapes and other visual stimuli that vary along such physical dimensions as brightness, shape, and size. These stimuli are popular in theory building because their characteristics are clearly specifiable and easily manipulated. However, as Haygood (1966) has pointed out, these stimuli are artificial and surely atypical of the kinds of stimuli

This research was reported in part at the meeting of the Southeastern Psychological Association, March 1981, Atlanta, Georgia. Requests for reprints may be addressed to Paul S. Siegel, Department of Psychology, P.O. Box 2968, The University of Alabama, University, Alabama 35486. normally encountered by human beings in their everyday ongoing behavior. This criticism reflects the growing concern of many over what has been described as the absence of "ecological validity" in our psychological experiments (Gibson, 1961; Neisser, 1976). Haygood suggested that verbal concepts offer a far more meaningful and more strongly representative stimulus domain for human subjects. He does, however, recognize difficulties associated with the use of verbal stimuli: First, words are generally described in terms of dichotomous categories (animal vs. fruit) rather than as positions along some continuous dimension; second, a related problem is that the dimensionality of verbal stimuli is difficult to specify; and third, words usually carry idiosyncratic affective or associational meaning. There is at least one additional problem: Even if the dimensionality of the verbal material can be specified, precise ordering or quantification of a word along that dimension is never easy, especially when compared with the relative ease with which physical stimuli may be quantified along such dimensions as volume, tone, size, and brightness.

Haygood (1966) proposed that these problems may be at least partially solved by utilizing the semantic dimensions developed by Osgood and his associates (Osgood, Suci, \& Tannenbaum, 1957). Although Haygood was concerned primarily with concept identification, his recommendations are equally appropriate in the present context. The Osgood bipolar dimensions of evaluation, activity, and potency seem, at least on an intuitive level, to closely parallel the physical dimensions commonly manipulated in discrimination learning studies.

Haygood (1966) established the feasibility of using the semantic differential dimensions in concept-learning studies. His subjects learned to categorize words based 
on these dimensions more efficiently than could be accounted for by rote learning. A major purpose of the present study was that of determining whether the semantic differential dimensions provide effective information to the human learner in a simultaneous, two-choice discrimination learning task.

As noted, the RRC facilitation effect is a primary finding in the discrimination learning literature. Considerable theoretical advance attends any extension of the boundary conditions. Exclusive dependence of the effect on visual stimuli greatly reduces theoretical generality. And so, the present research adopted as another goal the assessment of RRC facilitation as it might obtain with stimuli differing along semantic or conceptual dimensions.

\section{METHOD}

\section{Subjects}

Twenty-four college freshmen, recruited from classes in introductory psychology, served as volunteer subjects. Potential influences of gender and race were controlled through random assignment to groups.

\section{Apparatus}

A TRS-80 color computer (16K, extended BASIC) was used for the experiment. Instructions, as well as experimental stimuli, were presented on the screen, and subjects responded via the keyboard.

\section{Procedure}

Osgood's factor analyses and preliminary work in our laboratory have both suggested that "evaluation" is such a highly salient dimension that it may overshadow differences produced by other experimental manipulations. That is, if the evaluation dimension is relevant in a discrimination task, the subject's response will be based almost entirely on the "good-bad" distinction, regardless of other cues; the presence of a second, redundant dimension will exercise minimal effect. For this reason, the evaluation dimension was excluded from the present design. Activity and potency, on the other hand, appear to be weaker and roughly equal in salience. We chose to use those two dimensions exclusively.

Heise (1965) has developed a dictionary of 1,000 frequently occurring English words and presented their factor scores on each of Osgood's three dimensions. From this dictionary, we constructed three lists of 40 pairs each. Table 1 provides examples of pairs from each of these lists.

The first list, activity, consisted of word pairs that differed only in their factor scores on the activity dimension; one word was "active" and the other, "passive." These words were closely matched in terms of their factor scores on the other two dimensions, evaluation and potency. The second list, potency, was similarly constructed, but the words differed only in potency; one word was "strong" and the other, "weak." The third list, A\&P-matched, combined the activity and potency dimensions as redundant relevant cues. That is, one word was both active and strong, whereas the other was both passive and weak.

Heise's (1965) factor scores for each dimension yield a mean of 0 and a standard deviation of 1.00. Any two scores differing by more than 1.00 are considered reliably different. We originally required that the two words of each pair be separated by at least 2.00 factor scores on the relevant dimension(s). This rather strict requirement allowed us to extract lists of only 12 pairs each from Heise's dictionary. A pilot study revealed that short lists do not provide adequate sensitivity of measurement. Thus, we were forced to relax our inclusion
Table 1

Sample Pairs From Each Condition

\begin{tabular}{|c|c|c|}
\hline & & Cue Type \\
\hline \multicolumn{3}{|c|}{ Activity } \\
\hline 1. Drive & (He drove there) & Active \\
\hline 2. Listen & (He listened to it) & Passive \\
\hline 1. Former & (That was in former times) & Passive \\
\hline 2. Group & (He is in that group) & Active \\
\hline 1. Loss & (It is a loss) & Passive \\
\hline 2. Cut & (He cut it) & Active \\
\hline \multicolumn{3}{|c|}{ Potency } \\
\hline 1. Mean & (It meant this) & Weak \\
\hline 2. Degree & (It is to that degree) & Strong \\
\hline 1. Attempt & (It was his own attempt) & Strong \\
\hline 2. Movement & (There is movement there) & Weak \\
\hline 1. Oil & (It is oil) & Strong \\
\hline 2. Adopt & (He adopted their ideas) & Weak \\
\hline \multicolumn{3}{|c|}{ A\&P-Matched } \\
\hline 1. Duty & (He is duty-bound) & Active-Strong \\
\hline 2. Still & (He is still there) & Passive-Weak \\
\hline 1. Small & (It is small) & Passive-Weak \\
\hline 2. Control & (He has control of it) & Active-Strong \\
\hline 1. Lose & (He lost it) & Passive-Weak \\
\hline 2. Dangerous & (That is dangerous) & Active-Strong \\
\hline
\end{tabular}

criteria in order to obtain longer lists. For a word pair to be included in the present study, it was required that the two words be separated by at least 1.5 factor scores on the relevant dimen$\operatorname{sion}(\mathrm{s})$, and they were required to match within .20 factor scores on the irrelevant dimension(s). In addition, it was required that each word be separated from 0 (on relevant dimensions) by at least .75 units in the appropriate direction. Application of these criteria generated lists of 40 pairs in each of the three conditions. While some words occurred on more than one list, no word was repeated within a list.

Subjects were assigned randomly to the three groups (eight subjects per group) and tested singly. After responding to a sample pair to gain familiarity with the keyboard, all subjects were given the following instructions (via the computer screen).

"On this practice example the difference between the words was very simple-one was an animal and the other a fruit (dog and pear).

"The difference between the following words is not as simple. It has nothing to do with fruits and animals or anything like that. There are many other ways in which words can differ. We are concerned here with three particular ways. First, the word may carry along with it a 'good' or 'bad' feeling. Example-'succeed' versus 'terrible.' Think about this for a moment. Second, words may differ in 'activity,' such as 'run' versus 'sleep.' Think about this for a moment. Third, words may seem to convey 'strength' or 'weakness,' such as 'powerful' versus 'kitten.' Think about this for a moment.

"We are only concerned, then, with three characteristics: (1) good versus bad; (2) active versus passive; (3) strong versus weak. These characteristics apply to the general feeling you get from the word. On the first pair you will have no idea which word is correct. But as we go on, you may begin to see a pattern of which words are right and which are wrong. Also, the sentences under the words have nothing to do with which word is correct. Only the words themselves matter."

Word pairs were then presented in a vertical arrangement, one above the other. Under each word was its defining sentence, taken verbatim from Heise's (1965) dictionary. Timing was self-paced; subjects were allowed to study a pair as long as they wished before choosing. Immediately after the subject chose 
(by pressing Key 1 or Key 2), the appropriate feedback, "right" or "wrong," was displayed on the computer screen beside the word the subject had chosen. Differential auditory feedback was also provided. Subjects were then allowed to study the words and the feedback for as long as they wished before the next pair appeared (presentation was controlled by the subject).

The number of correct responses, of 40, was recorded for each subject. Data were analyzed through univariate one-way ANOVA.

\section{RESULTS}

The treatment effect was significant $[F(2,21)=11.12$, $\mathrm{p}<.01]$. Eta squared indicated that the treatment accounted for about $51 \%$ of the variance (.514). Student Newman-Keuls multiple-comparison tests revealed that the redundant cue condition, A\&P-matched, produced a significantly larger number of correct responses than did either of the single-cue conditions (activity and potency); the single-cue arrangements did not differ from each other. Group means were as follows: activity $=23.38$, potency $=22.13$, and A\&P-matched $=30.75$.

\section{DISCUSSION}

We are now able to answer in the affirmative our original questions. The semantic differential dimensions do indeed provide adequate information to the learner in a simultaneous two-choice discrimination learning task. And, clearly, the RRC facilitatory effect holds true for this stimulus domain.

The present results invite additional research with the semantic differential dimensions, perhaps to explore other phenomena prominent in the discrimination learning literature. Intra- and extradimensional shifts and the overlearning reversal effect (ORE) are prime candidates. The stimulus domain examined here may provide a new and more nearly ecologically valid arena for comparative testing of particular theories of discrimination learning.

\section{REFERENCES}

Bourne, L. E., JR., \& Haygood, R. C. The role of stimulus redundancy in concept identification. Journal of Experimental Psychology, 1959, 58, 232-238.

Fisher, M. A., \& Zeaman, D. An attention-retention theory of retardate discrimination learning. In N. R. Ellis (Ed.), International review of research in mental retardation (Vol. 6). New York: Academic Press, 1973.

Gibson, J. J. Ecological optics. Vision Research, 1961, 1, 253-262.

HAYGOOD, R. C. Use of semantic differential dimensions in concept learning. Psychonomic Science, 1966, 5, 305-306.

Heise, D. R. Semantic differential profiles for 1,000 most frequent English words. Psychological Monographs, 1965, 601, 1-31.

Kendler, H. H., \& Kendler, T. S. Vertical and horizontal processes in problem solving. Psychological Review, 1962, 69, 1-16.

Neisser, U. Cognition and reality. San Francisco: Freeman, 1976.

Osgood, C. E., Suci, G. J., \& Tannenbaum, P. H. The measurement of meaning. Urbana: University of Illinois Press, 1957.

Sutherland, N. S., \& Mackintosh, N. J. Mechanisms of animal discrimination learning. New York: Academic Press, 1971.

Trabasso, T., \& Bower, G. H. Attention in learning: Theory and research. New York: Wiley, 1968.

(Received for publication June 12, 1981.) 